\title{
Effect of viscous dissipation on mixed convection flow in a vertical double passage channel using Robin boundary conditions
}

\author{
J. Prathap Kumar ${ }^{1}$, J.C. Umavathi ${ }^{2 *}$ and M. Karuna Prasad ${ }^{1}$ \\ ${ }^{1}$ Department of Mathematics, Gulbarga University, Kalaburagi-585 106, Karnataka, INDIA \\ ${ }^{2}$ Department of Engineering, University of Sannio, Piazza Roma 21, 82100 Benevento, ITALY \\ *Corresponding author E-mail: drumavathi@ rediffmail.com
}

\begin{abstract}
The laminar fully developed flow in a vertical double passage channel filled with clear fluid has been discussed using Robin boundary conditions. The thin perfectly conductive baffle is inserted in the channel. The governing equations of the fluid which are coupled and nonlinear are solved analytically by the perturbation method and semi analytically using differential transform method (DTM). The reference temperature of the external fluid is considered to be equal and different. The perturbation method which is valid for small values of perturbation parameter is used to find the combined effects of buoyancy forces and viscous dissipation. The limitation imposed on the perturbation parameter is relaxed by solving the basic equations using differential transform method. The influence of mixed convection parameter, Biot number for symmetric and asymmetric wall temperatures on the velocity, temperature and the Nusselt number is explored at different positions of the baffle. The solutions obtained by differential transform method are justified by comparing with the solutions obtained by perturbation method and the solutions agree very well for small values of the perturbation parameter.
\end{abstract}

Keywords: Baffle, Differential Transform Method, Perturbation Method, Viscous dissipation, Robin Boundary Conditions, Double passage channel.

DOI: http://dx.doi.org/10.4314/ijest.v8i3.4

\section{Introduction}

Mixed convection in ducted flow may occur in many applications, such as in heat exchangers, chemical processing equipment and transport of heated or cooled fluids, solar collectors and microelectronic cooling. When the buoyancy opposes or aids the forced flow the flow reversal is observed near the duct wall. These flow reversals are of substantial significance because they may strongly affect the wall friction, pressure drop, heat transfer, occurrence of entrance temperature, and stability of flow. Several papers on mixed convection in a parallel-plate vertical channel are available in the literature. Aung and Worku (1986) studied the boundary conditions of uniform wall temperatures. The effect of viscous dissipation on the velocity and on the temperature profiles has been analysed by Barletta (1998) for the boundary conditions of uniform wall temperatures. Mixed convection in a vertical channel was also studied by Umavathi and her group (2006, 2009, 2010, 2011, 2013).

Attaching baffles to its vertical wall or horizontal walls partitions the enclosure. The flow characteristics of partitioned cavity are studied recently by both numerical and experimental methods. Mixed convection flow through square vented enclosure with partially heated bottom wall is often encountered in air-condition system. Air heating is one of the preferred methods for conditioning the room space especially in cold countries. With the increasing living standard, people pay special attention to the indoor air quality (IAQ), which is essential for the health of the people and work efficiency. To ensure better IAQ, a new air conditioning system with localized heat source and displacement ventilation has been proposed. The interaction between natural convection and forced convection is the main theme for displacement ventilation system. Mixed convection flow in an enclosure 
was studied by Angirasa (2000) using isothermal wall conditions. Free and forced convection from a flush-mounted heat source on the bottom of a horizontal rectangular enclosure was studied by Sumon Saha et al. (2006).

Salah-El-Din (1994) studied mixed convection problem in a vertical double passage channel. He claimed that the presence of the baffle will lead to a higher value of Nusselt number depending on the baffle position and the value of the ratio of Grashof number to Reynolds number. Experimental results by Datta and Datta (1998) also proved the enhancement of heat transfer with inclined solid and perforated baffles including the effects of baffle size, position and orientation. The experimental results by Chen and Chen (1998) showed that the local heat transfer co-efficient decreases with an increase in the baffle wall gap for small baffle widths whereas for large baffle widths the heat transfer co-efficient was increased. The effect of horizontal baffle on the heat transfer characteristics of opposing mixed convection in a vertical open channel was studied by Chang and Shiau (2005). Mixed convection in a vertical channel saturated with porous medium was studied by Umavathi et al. (2014) in the presence of baffle and they found that by increasing the porous parameter, rate of heat transfer was enhanced at both the walls. Prathap Kumar et al. (2011a,b) and Umavathi (2011) also studied convection in a vertical channel using different fluids by introducing a baffle.

In the literature we find that forced convection heat transfer in the thermal entrance region for a rectangular channel was studied for temperature boundary condition of first kind for prescribed wall temperature (Javeri, 1976), and for second kind for prescribed wall heat flux (Sparrow, 1960). However temperature boundary condition of third kind (the local wall heat flux is a linear function of the local wall temperature) is more realistic in real field applications. Javeri $(1977,1978)$ studied the boundary condition of third kind for flow over a flat plate and in the thermal entrance region of a rectangular channel. The effect of viscous dissipation on mixed convection in a vertical channel using Robin boundary conditions was also studied by Zanchini (1998). Following the work of Zanchini (1998), Umavathi and Santosh (2012a,b) and Umavathi and Jaweria (2011a,b) and Umavathi et al. (2012) studied mixed convection in a vertical channel using Robin boundary conditions. Recently the influence of baffle for natural convection in a rectangular enclosure was studied by Sivasankaran and Kandaswamy (2007). Using power-law liquid, Hajmohammadi and Nourazar (2014) studied the effect of a thin gas layer in micro cylindrical Couette flows. The aim of this paper is to extend the analysis performed by Zanchini (1998) by inserting perfectly conducting baffle using Robin boundary conditions.

\section{Formulation of the problem}

Figure 1 displays the schematic diagram and the co-ordinates of the problem. The flow is assumed to be steady, laminar and fully developed. The perfectly thin baffle is inserted in the channel and hence we obtain two passages.

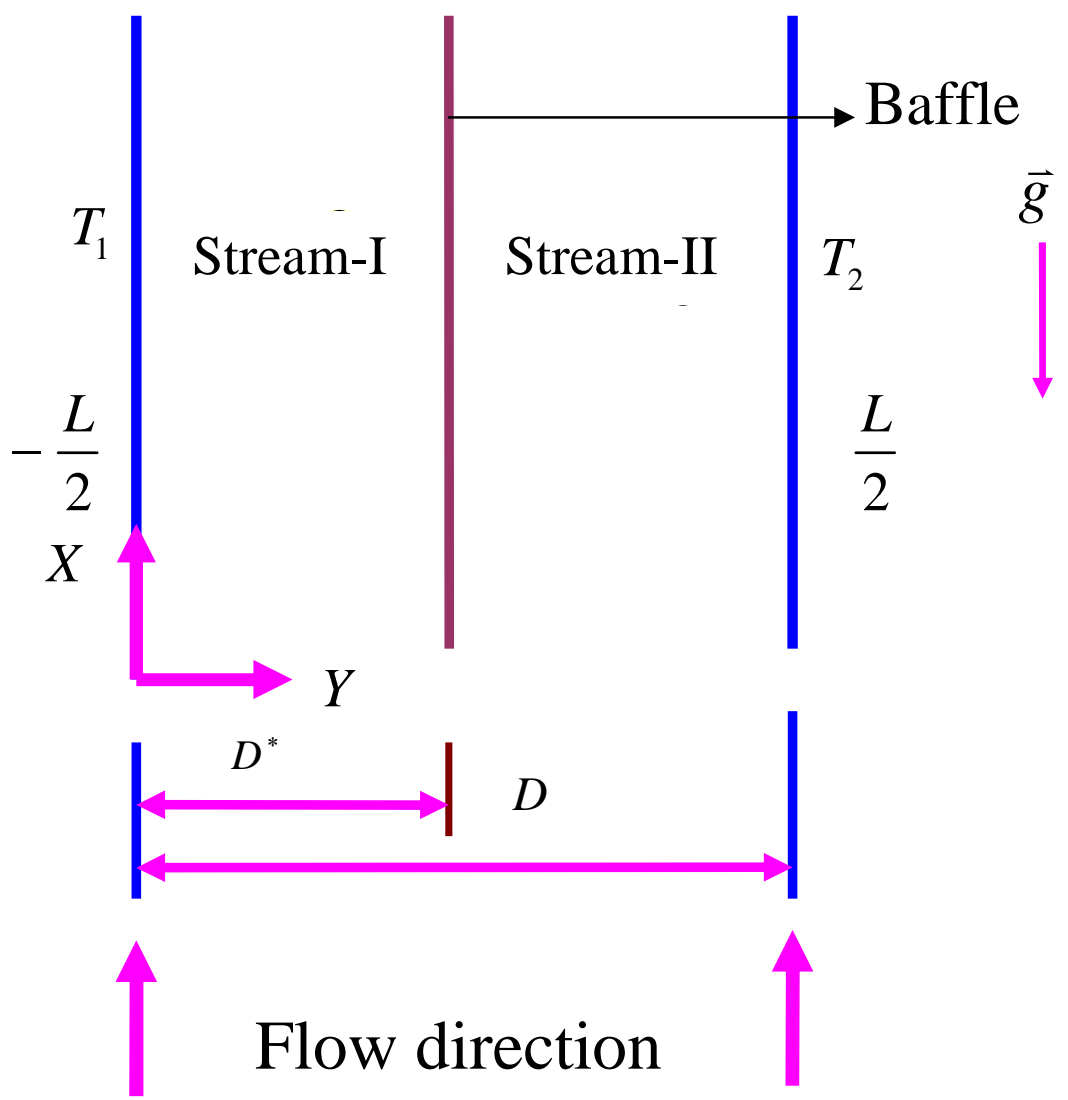

Figure 1: Schematic diagram and the coordinate system 
The physical properties such as thermal conductivity and viscosity are assumed to be constant. Density is assumed to be constant except when it is multiplied by the gravity and state equation also holds. The Navier-Stokes equations using the assumptions as mentioned above (following Zanzchini, 1998) become

Stream-I

$$
\rho \beta g\left(T_{1}-T_{0}\right)-\frac{\partial P}{\partial X}+\mu \frac{d^{2} U_{1}}{d Y^{2}}=0
$$

Stream-II

$$
\rho \beta g\left(T_{2}-T_{0}\right)-\frac{\partial P}{\partial X}+\mu \frac{d^{2} U_{2}}{d Y^{2}}=0
$$

and the momentum balance equation along $Y$ direction in both the streams are

$$
\frac{\partial P}{\partial Y}=0
$$

where

$$
P=p+\rho_{0} g X
$$

(assuming equal pressure gradient in both the streams) is the difference between the pressure and the hydrostatic pressure. Equations (1) and (2) using Equation (4) become

Stream-I

$$
\rho \beta g\left(T_{1}-T_{0}\right)-\frac{d P}{d X}+\mu \frac{d^{2} U_{1}}{d Y^{2}}=0
$$

Stream-II

$$
\rho \beta g\left(T_{2}-T_{0}\right)-\frac{d P}{d X}+\mu \frac{d^{2} U_{2}}{d Y^{2}}=0
$$

Differentiating Equations (5) and (6) with respect to $X$ and $Y$, one obtains

Stream-I

$$
\begin{aligned}
& \frac{\partial T_{1}}{\partial X}=\frac{1}{\rho \beta g} \frac{d^{2} P}{d X^{2}} \\
& \frac{\partial T_{1}}{\partial Y}=-\frac{\mu}{\rho \beta g} \frac{d^{3} U_{1}}{d Y^{3}} \\
& \frac{\partial^{2} T_{1}}{\partial Y^{2}}=-\frac{\mu}{\rho \beta g} \frac{d^{4} U_{1}}{d Y^{4}}
\end{aligned}
$$

Stream-II

$\frac{\partial T_{2}}{\partial X}=\frac{1}{\rho \beta g} \frac{d^{2} P}{d X^{2}}$

$\frac{\partial T_{2}}{\partial Y}=-\frac{\mu}{\rho \beta g} \frac{d^{3} U_{2}}{d Y^{3}}$

$\frac{\partial^{2} T_{2}}{\partial Y^{2}}=-\frac{\mu}{\rho \beta g} \frac{d^{4} U_{2}}{d Y^{4}}$

It is assumed that the thickness of the walls is negligible and exchange heat by convection with an external fluid. The external convection coefficient at the left wall is $h_{1}$ and at the right wall is $h_{2}$. It is also assumed that the temperature at the right wall is greater than or equal to the temperature of the left wall. The boundary conditions on the temperature field are

$$
\begin{aligned}
& -\left.k \frac{\partial T_{1}}{\partial Y}\right|_{Y=-\frac{L}{2}}=h_{1}\left[T_{1}-T(X,-L / 2)\right] \\
& -\left.k \frac{\partial T_{2}}{\partial Y}\right|_{Y=\frac{L}{2}}=h_{2}\left[T(X, L / 2)-T_{2}\right]
\end{aligned}
$$


Using Eqns. (8) and (11), Eqns.(13) and (14) become

$$
\begin{aligned}
& \left.\frac{d^{3} U_{1}}{d Y^{3}}\right|_{Y=-\frac{L}{2}}=\frac{\rho \beta g h_{1}}{\mu k}\left[T_{1}-T(X,-L / 2)\right] \\
& \left.\frac{d^{3} U_{2}}{d Y^{3}}\right|_{Y=\frac{L}{2}}=\frac{\rho \beta g h_{2}}{\mu k}\left[T(X, L / 2)-T_{2}\right]
\end{aligned}
$$

Equations (7) and (10) suggest that there exists a constant $A$ such that

$$
\frac{d P}{d X}=A
$$

The equation for the conservation of energy in the two passages are

Stream-I

$\frac{d^{2} T_{1}}{d Y^{2}}=-\frac{\mu}{k}\left(\frac{d U_{1}}{d Y}\right)^{2}$

Stream-II

$\frac{d^{2} T_{2}}{d Y^{2}}=-\frac{\mu}{k}\left(\frac{d U_{2}}{d Y}\right)^{2}$

Equations (9) and (18) and Eqns. (12) and (19) yield differential equations for $U_{1}$ and $U_{2}$ as follows

Stream-I

$\frac{d^{4} U_{1}}{d Y^{4}}=\frac{\rho \beta g}{k}\left(\frac{d U_{1}}{d Y}\right)^{2}$

Stream-II

$\frac{d^{4} U_{2}}{d Y^{4}}=\frac{\rho \beta g}{k}\left(\frac{d U_{2}}{d Y}\right)^{2}$

The boundary conditions for the velocity in stream-I and stream-II are

$U_{1}\left(-\frac{L}{2}\right)=U_{2}\left(\frac{L}{2}\right)=0$

and together with Eqs. (15) and (16), which on account of (5) and (6) can be written as

$\left[\frac{d^{3} U_{1}}{d Y^{3}}-\frac{h_{1}}{k} \frac{d^{2} U_{1}}{d Y^{2}}\right]_{Y=-L / 2}=-\frac{A h_{1}}{\mu k}-\frac{\rho \beta g h_{1}}{\mu k}\left(T_{0}-T_{1}\right)$

$\left[\frac{d^{3} U_{2}}{d Y^{3}}+\frac{h_{2}}{k} \frac{d^{2} U_{2}}{d Y^{2}}\right]_{Y=L / 2}=\frac{A h_{2}}{\mu k}-\frac{\rho \beta g h_{2}}{\mu k}\left(T_{2}-T_{0}\right)$

In addition we consider the continuity of temperature $\left(T_{1}=T_{2}\right)$ and continuity of heat flux $\left(\frac{d T_{1}}{d Y}=\frac{d T_{2}}{d Y}\right)$ at the baffle position.

Applying these conditions in Eqns. (5), (6), (8) and (11) yield

$\frac{d^{2} U_{1}}{d Y^{2}}=\frac{d^{2} U_{2}}{d Y^{2}}$

and

$\frac{d^{3} U_{1}}{d Y^{3}}=\frac{d^{3} U_{2}}{d Y^{3}}$

Equations (20) and (21) which determine the velocity distribution along with the boundary conditions at the channel walls and at the baffle position are non-dimensionalised using the following dimensionless parameters: 


$$
\begin{aligned}
& u_{i}=\frac{U_{i}}{U_{0}}, y=\frac{Y}{D}, y^{*}=\frac{Y^{*}}{D^{*}}, G r=\frac{g \beta \Delta T D^{3}}{v^{2}}, \operatorname{Re}=\frac{U_{0} D}{v}, B r=\frac{U_{0}^{2} \mu}{k \Delta T}, v=\frac{\mu}{\rho}, \Lambda=\frac{G r}{\operatorname{Re}} \quad \theta=\frac{T_{i}-T_{0}}{\Delta T}, \\
& B i_{1}=\frac{h_{1} D}{k}, B i_{2}=\frac{h_{2} D}{k}, S=\frac{B i_{1} B i_{2}}{B i_{1} B i_{2}+2 B i_{1}+2 B i_{2}}
\end{aligned}
$$

In the above equation $D=2 L$ is the hydraulic diameter, and the reference velocity and the reference temperature are given by

$U_{0}=-\frac{A D^{2}}{48 \mu} \quad T_{0}=\frac{T_{1}+T_{2}}{2}+S\left(\frac{1}{B i_{1}}-\frac{1}{B i_{2}}\right)\left(T_{2}-T_{1}\right)$

As in Barletta (1998) the reference temperature $\Delta T$ is given either by

$\Delta T=T_{2}-T_{1}$ if $T_{1}<T_{2}$

or by

$$
\Delta T=\frac{\mu^{2}}{\rho^{2} C_{p} D^{2}} \quad \text { if } \quad T_{1}=T_{2}
$$

Therefore the value of the dimensionless parameter $R_{T}$ can be either 0 or 1 . For asymmetric heating $\left(T_{1}<T_{2}\right) R_{T}$ is equal to one and will be zero for symmetric heating $\left(T_{1}=T_{2}\right)$. Equation (17) implies that $A$ can be either positive or negative. If $A<0$, then $U_{0}, \operatorname{Re}$ and $\Lambda$ are positive, and the flow is upward. On the other hand, if $A>0$, the flow is downward, so that $U_{0}, \operatorname{Re}$ and $\Lambda$ are negative.

Stream-I

By employing the dimensionless quantities as defined in Eq. (27), Eqns. (20)-(26) can be written as

$$
\frac{d^{4} u_{1}}{d y^{4}}=\Lambda B r\left(\frac{d u_{1}}{d y}\right)^{2}
$$

Stream-II

$$
\begin{gathered}
\frac{d^{4} u_{2}}{d y^{4}}=\Lambda B r\left(\frac{d u_{2}}{d y}\right)^{2} \\
u_{1}=0 ; \quad \frac{d^{2} u_{1}}{d y^{2}}-\frac{1}{B i_{1}} \frac{d^{3} u_{1}}{d y^{3}}=-48+\frac{R_{T} \Lambda S}{2}\left(1+\frac{4}{B i_{1}}\right) \quad \text { at } \quad y=-\frac{1}{4} \\
u_{2}=0 ; \quad \frac{d^{2} u_{2}}{d y^{2}}+\frac{1}{B i_{2}} \frac{d^{3} u_{2}}{d y^{3}}=-48-\frac{R_{T} \Lambda S}{2}\left(1+\frac{4}{B i_{2}}\right) \text { at } \quad y=\frac{1}{4} \\
u_{1}=0 ; \quad u_{2}=0 ; \quad \frac{d^{2} u_{1}}{d y^{2}}=\frac{d^{2} u_{2}}{d y^{2}} ; \quad \frac{d^{3} u_{1}}{d y^{3}}=\frac{d^{3} u_{2}}{d y^{3}} ; \quad \text { at } \quad y=y^{*} .
\end{gathered}
$$

\section{Solutions Using Perturbation Method}

Equations (31) and (32) are nonlinear and coupled differential equations. Hence finding closed form solutions can not be found. However using regular perturbation method approximate solutions can be found. The product of mixed convection parameter and Brinkman number is chosen as the perturbation parameter. Therefore the expressions for the velocity and temperature can be written as

$$
u_{i}(y)=u_{i 0}(y)+\varepsilon u_{i 1}(y)+\varepsilon^{2} u_{i 2}(y)+\ldots
$$

Substituting Eqn. (34) into Eqns. (31), (32) and (33) and equating the coefficients of like power of $\varepsilon$ to zero and one, the zeroth and first order equations become

Stream-I

Zeroth order equations 


$$
\frac{d^{4} u_{10}}{d y^{4}}=0
$$

First order equations

$$
\frac{d^{4} u_{11}}{d y^{4}}=\left(\frac{d u_{10}}{d y}\right)^{2}
$$

Stream-II

Zeroth order equations

$$
\frac{d^{4} u_{20}}{d y^{4}}=0
$$

First order equations

$$
\frac{d^{4} u_{21}}{d y^{4}}=\left(\frac{d u_{20}}{d y}\right)^{2}
$$

The corresponding boundary conditions for both stream-I and stream-II are

Zeroth order

$$
\begin{array}{ll}
u_{10}=0 ; \quad \frac{d^{2} u_{10}}{d y^{2}}-\frac{1}{B i_{1}} \frac{d^{3} u_{10}}{d y^{3}}=B c_{1} \quad \text { at } \quad y=-\frac{1}{4} \\
u_{20}=0 ; \quad \frac{d^{2} u_{20}}{d y^{2}}+\frac{1}{B i_{2}} \frac{d^{3} u_{20}}{d y^{3}}=B c_{2} \quad \text { at } \quad y=\frac{1}{4} \\
u_{10}=0 ; \quad u_{20}=0 ; \quad \frac{d^{2} u_{10}}{d y^{2}}=\frac{d^{2} u_{20}}{d y^{2}} ; \quad \frac{d^{3} u_{10}}{d y^{3}}=\frac{d^{3} u_{20}}{d y^{3}} \quad \text { at } y=y^{*}
\end{array}
$$

where

$$
B c_{1}=-48+\frac{\Lambda R_{T} s}{2}\left(1+\frac{4}{B i_{1}}\right) \text { and } B c_{2}=-48-\frac{\Lambda R_{T} s}{2}\left(1+\frac{4}{B i_{2}}\right)
$$

First order

$$
\begin{array}{llrl}
u_{11}=0 ; & \frac{d^{2} u_{11}}{d y^{2}}-\frac{1}{B i_{1}} \frac{d^{3} u_{11}}{d y^{3}}=0 & \text { at } \quad y=-\frac{1}{4} \\
u_{21}=0 ; & \frac{d^{2} u_{21}}{d y^{2}}+\frac{1}{B i_{2}} \frac{d^{3} u_{21}}{d y^{3}}=0 & \text { at } \quad y=\frac{1}{4} \\
u_{11}=0 ; & u_{21}=0 ; \quad \frac{d^{2} u_{11}}{d y^{2}}=\frac{d^{2} u_{21}}{d y^{2}} ; & \frac{d^{3} u_{11}}{d y^{3}}=\frac{d^{3} u_{21}}{d y^{3}} \quad \text { at } y=y^{*}
\end{array}
$$

The solutions of zeroth and first order Eqns. (35)-(38) using the boundary conditions (39) and (40) can be obtained and are not presented.

Nondimensionalisation of Eqs. (5) and (6) become

Stream-I

$$
\theta_{1}=-\frac{1}{\Lambda}\left(48+\frac{d^{2} u_{1}}{d y^{2}}\right)
$$

Stream-II

$$
\theta_{2}=-\frac{1}{\Lambda}\left(48+\frac{d^{2} u_{2}}{d y^{2}}\right)
$$

Using the solutions of the velocity field, the temperature field is computed. 


\section{Solutions Using Differential Transform Method}

The Differential Transform Method was first introduced by Zhou (1986) who solved linear and nonlinear differential equations in electric circuit analysis. This method constructs a semi-analytical numerical technique uses Taylor series expansion for the solution of linear or nonlinear partial or ordinary differential equations in the form of a polynomial.

The differential transform of the $k^{\text {th }}$ derivative of the function $f(y)$ is defined as follows:

$$
F[k]=\frac{1}{k !}\left[\frac{d^{k} f(y)}{d y^{k}}\right]_{y=y_{0}}
$$

where $f(y)$ is the original function and $F[k]$ is the transformed function. Differential inverse transform of $F[k]$ is defined as follows:

$$
f(y)=\sum_{k=0}^{\infty} F[k]\left(y-y_{0}\right)^{k}
$$

which implies that the concept of differential transform method is derived from Taylor series expansion, although this method is not able to evaluate the derivatives symbolically.

The Differential Transform Method (DTM) has been applied to obtain the solutions of equations (31) and (32) by using the boundary conditions obtained in Eqn. (33).

$$
\begin{aligned}
& (r+1)(r+2)(r+3)(r+4) u_{1}[r+4]=\Lambda B r \sum_{s=0}^{r}(r-s+1) u_{1}[r-s+1](s+1) u_{1}[s+1] \\
& (r+1)(r+2)(r+3)(r+4) u_{2}[r+4]=\Lambda B r \sum_{s=0}^{r}(r-s+1) u_{2}[r-s+1](s+1) u_{2}[s+1]
\end{aligned}
$$

The initial conditions are as follows

$$
u_{1}[0]=a 1, u_{1}[1]=a 2, u_{1}[2]=a 3, u_{1}[3]=a 4, u_{2}[0]=b 1, u_{2}[1]=b 2, u_{2}[2]=b 3, u_{2}[3]=b 4
$$

The numerical values of the constants a1, a2, a3, a4, b1, b2, b3, b4 can be find by using the transformed equations with boundary and interface conditions. The system of equations leads to the solutions by substituting obtained numerical values of constants.

\section{Nusselt Number}

The non-dimensional heat transfer coefficient known as Nusselt number is given by

$N u=\frac{d \theta}{d y}$

The Nusselt number at both left wall and right wall are calculated numerically for different values of several governing parameters and are given in the following Table 1.

Table 1: Values of Nusselt number at different positions of the baffle for

$$
\Lambda=500, B i_{1}=B i_{2}=10, R_{T}=1, \varepsilon=0.1
$$

\begin{tabular}{|c|c|c|c|c|c|c|}
\hline & \multicolumn{2}{|c|}{$\left.\frac{d \theta}{d y}\right|_{y=-0.25}$} & \multicolumn{3}{c|}{$\left.\frac{d \theta}{d y}\right|_{y=0.25}$} \\
\hline & & & & \\
& & & & & \\
\end{tabular}


Table 1 (cont'd): Values of Nusselt number at different positions of the baffle for $\Lambda=500, B i_{1}=B i_{2}=10, R_{T}=1, \varepsilon=0.1$

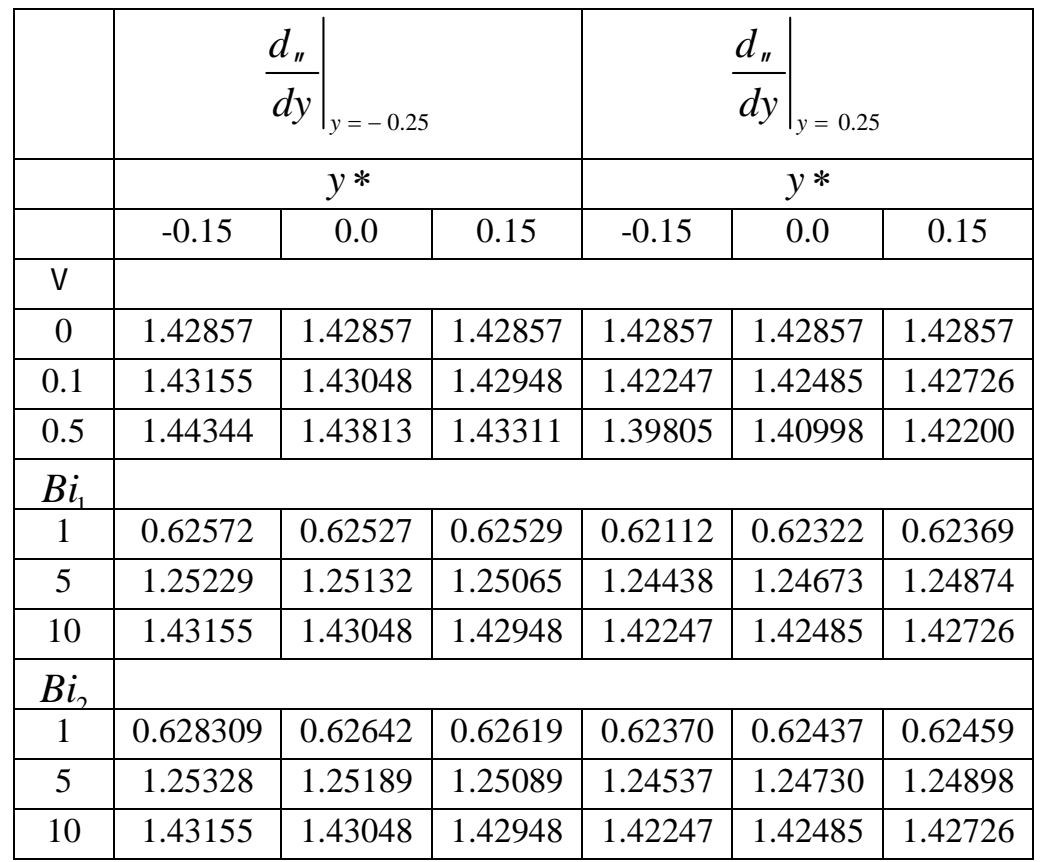

\section{Results and discussion}

In this paper, we have analyzed the problem of mixed convection flow in a vertical channel filled with purely viscous fluid using Robin boundary conditions by introducing a perfectly thin conducting baffle. The analytical solutions are obtained using regular perturbation method with the product of ratio of Grashof number to Reynolds number and Brinkman number as the perturbation parameter $\varepsilon(=B r(G r / R e))$ which is valid for small values of perturbation parameter. This restriction is relaxed by finding the solutions the using semi-numerical method known as differential transform method ((DTM). The combined effects of buoyancy and viscous dissipation on the momentum and heat transfer are analyzed and discussed for symmetric and asymmetric wall temperatures at different positions of the baffle. Figures 2 and 3 show the variations of the non-dimensional velocity and temperature fields for different values of mixed convection parameter $\Lambda$ for equal Biot numbers. For buoyancy assisting flow $(\Lambda>0)$, the velocity increases in stream-II, for buoyancy opposing flow $(\Lambda<0)$ and the velocity increases in stream-I as $\Lambda$ increases at all the baffle positions. As the mixed convection parameter $\Lambda$ increases, the reversal of flow increases at both the plates. Flow reversal is observed near the left wall (cold wall) for $\Lambda>0$ and near the right wall for $\Lambda<0$. This is because, the mixed convection parameter $\Lambda$ is the ratio of Grashof number to Reynolds number and therefore increasing $\Lambda$ implies increase of the buoyancy force and hence flow is enhanced for $\Lambda>0$ and retarded for $\Lambda<0$ at all the baffle positions. Figure 3 display the temperature profiles for different values of $\Lambda$ and it is seen that the temperature field is not much affected for the variations of $\Lambda$ at all the baffle positions. However, when the graph is enlarged it is seen that temperature increases in the upward direction for $\Lambda>0$ and in downward direction for $\Lambda<0$. The magnitude of temperature for buoyancy assisting flow is more when compare to buoyancy opposing flow.

Figures 4 and 5 are the plots of velocity and temperature fields for variations of $\Lambda$ for unequal Biot numbers for asymmetric wall heating. The effect of $\Lambda$ on the flow for unequal Biot numbers show the similar nature as that of equal Biot numbers except that the magnitude of flow reversal is less for unequal Biot numbers when compare to equal Biot numbers. This is due to the fact that, the value of $S$ becomes 339.5 for equal Biot numbers and the value of $S$ becomes 249.99 for unequal Biot numbers which implies that the convection is reduced for unequal Biot numbers. For unequal Biot numbers also the temperature field is not much affected by the variations of $\Lambda$. In order to know the effect of distribution of velocity and temperature for symmetric heating, the profiles are drawn for equal and unequal Biot numbers in Figures 6 to 9 at different baffle positions. It is seen from Figures 6 and 8 that the velocity field is not affected by the variations of $\Lambda$ at all the baffle positions for both equal and unequal Biot numbers. The magnitude of velocity field for buoyancy assisted $(\Lambda>0)$ flow is more when compared with buoyancy opposing $(\Lambda<0)$ flow. Further the optimal velocity is attained in stream-II when the baffle is near the left wall, in stream-II 
when the baffle is placed near the right wall and is symmetric when the baffle is positioned in the center of the channel. It is interesting to note that the mixed convection parameter $\Lambda$ is operative for symmetric wall heating when compared with asymmetric wall heating for both equal and unequal Biot numbers. One can also view that the temperature profiles look symmetric for equal Biot numbers and becomes stronger near the left plate for small values $B i_{1}$ at all the baffle positions. The value of $\varepsilon=0.1(\Lambda>0)$ and $\varepsilon=-0.1(\Lambda<0)$ in Figures 2 to 9 and the plots are drawn using perturbation method.
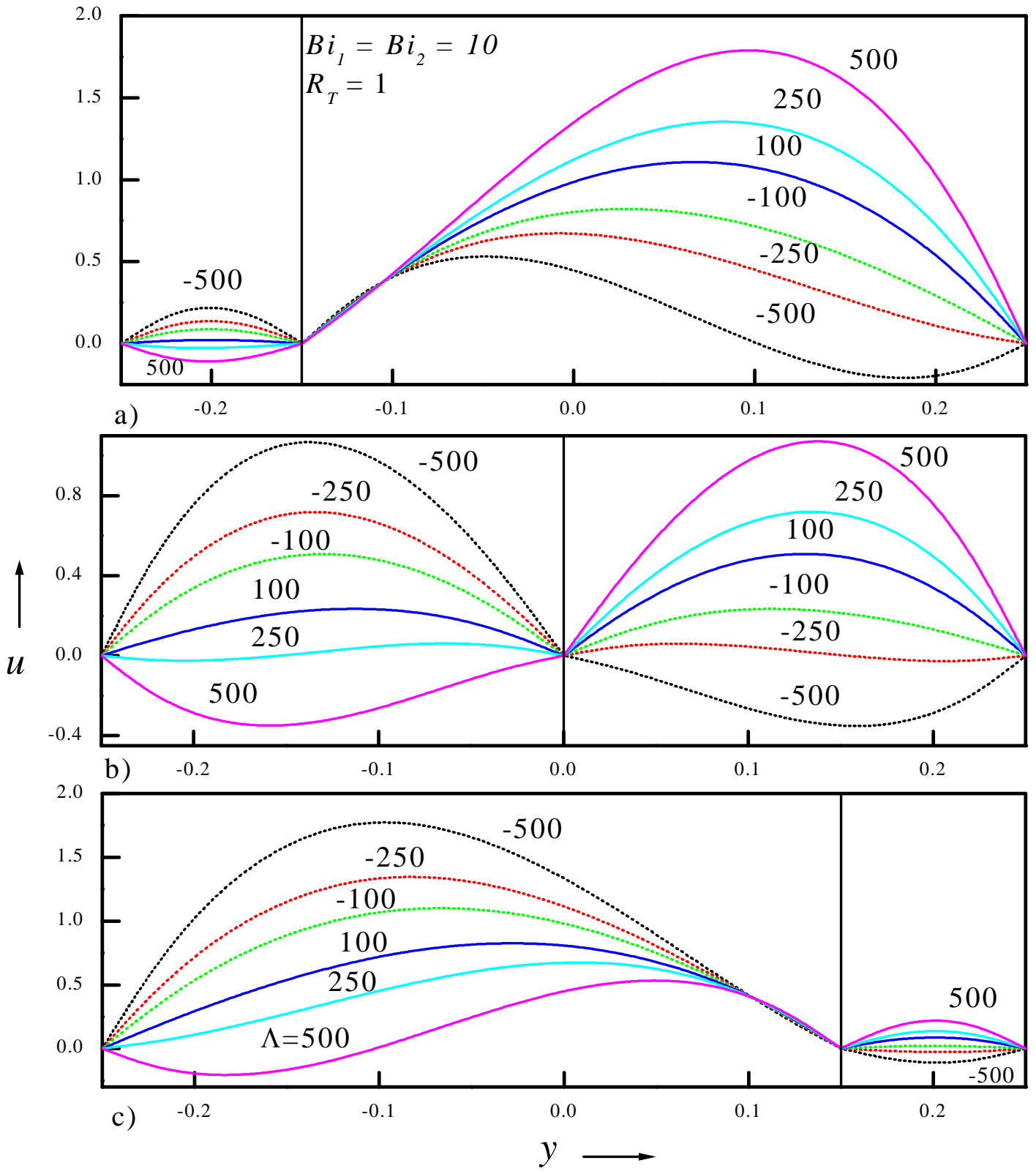

Figure 2: Velocity profiles for different values of $\Lambda$ at
a) $y^{*}=-0.15$,
b) $y^{*}=0.0$
c) $y^{*}=0.15$. 

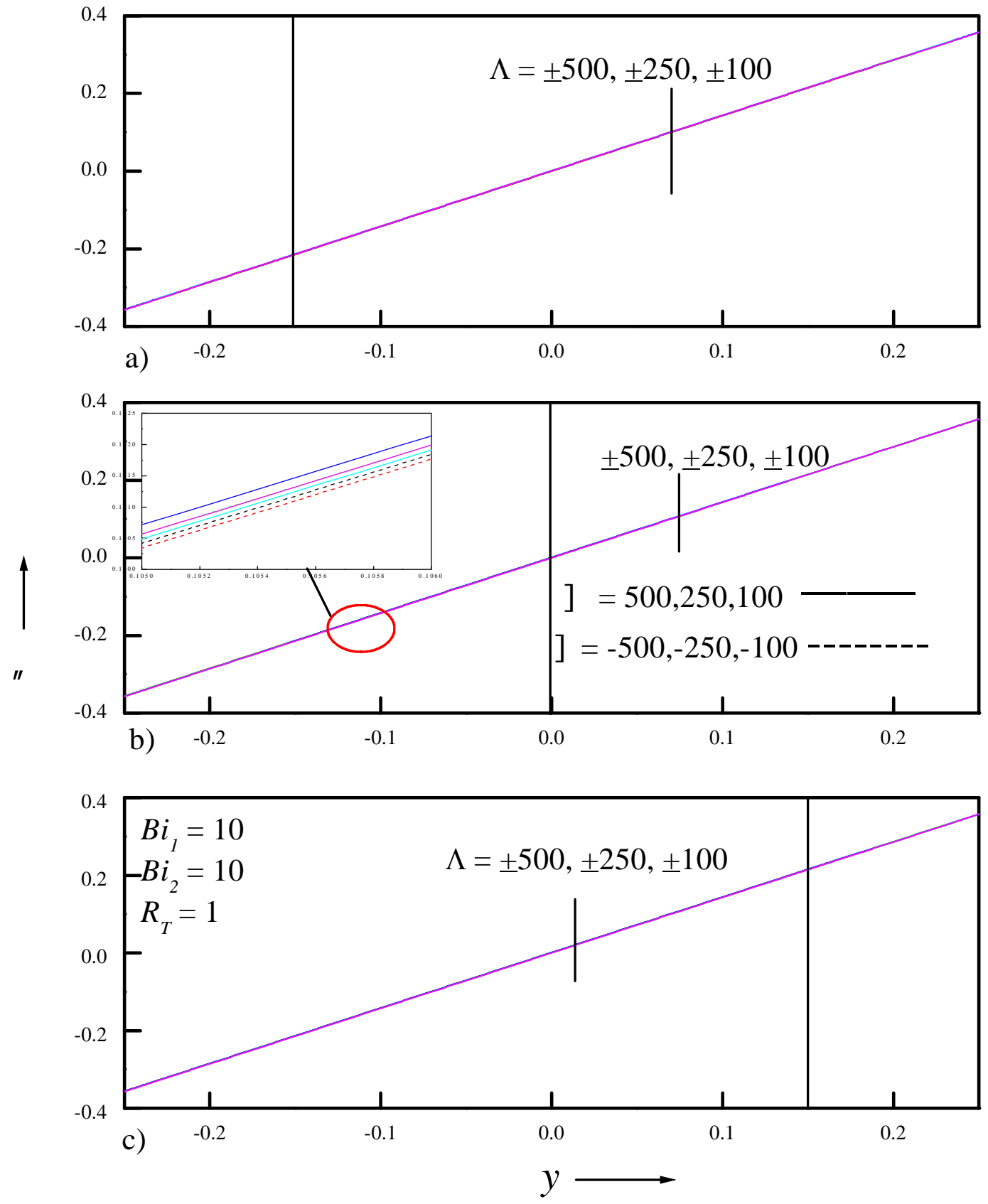

Figure 3: Temperature profiles for different values of $\Lambda$ at
a) $y^{*}=-0.15$,
b) $y^{*}=0.0$
c) $y^{*}=0.15$. 

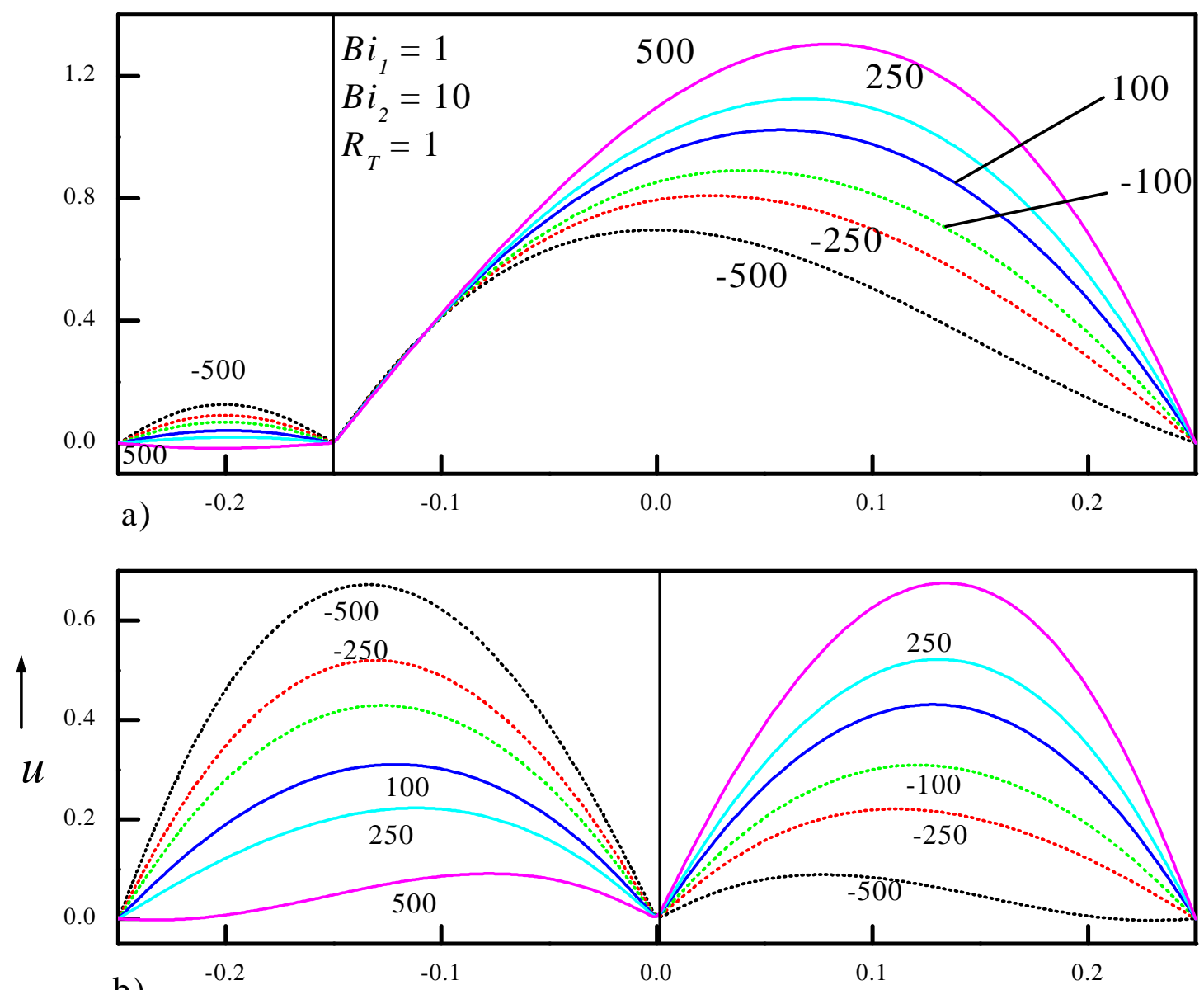

b)

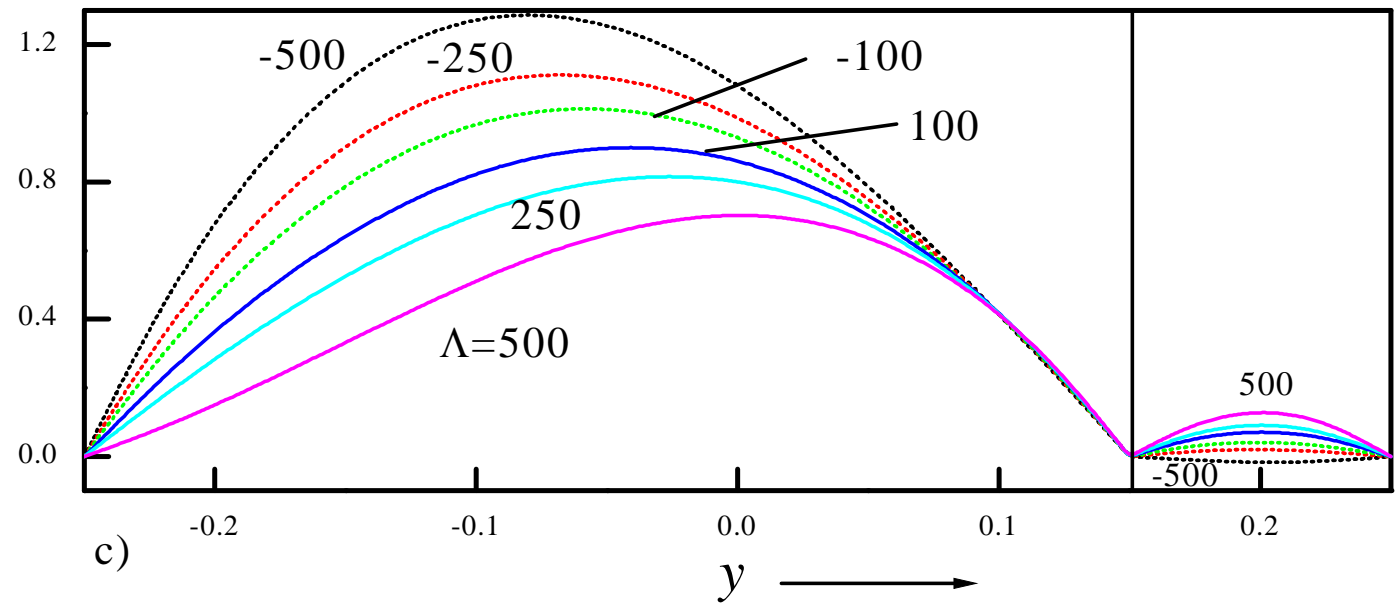

Figure 4: Velocity profiles for different values of $\Lambda$
a) $y^{*}=-0.15$,
b) $y^{*}=0.0$
c) $y^{*}=0.15$ 

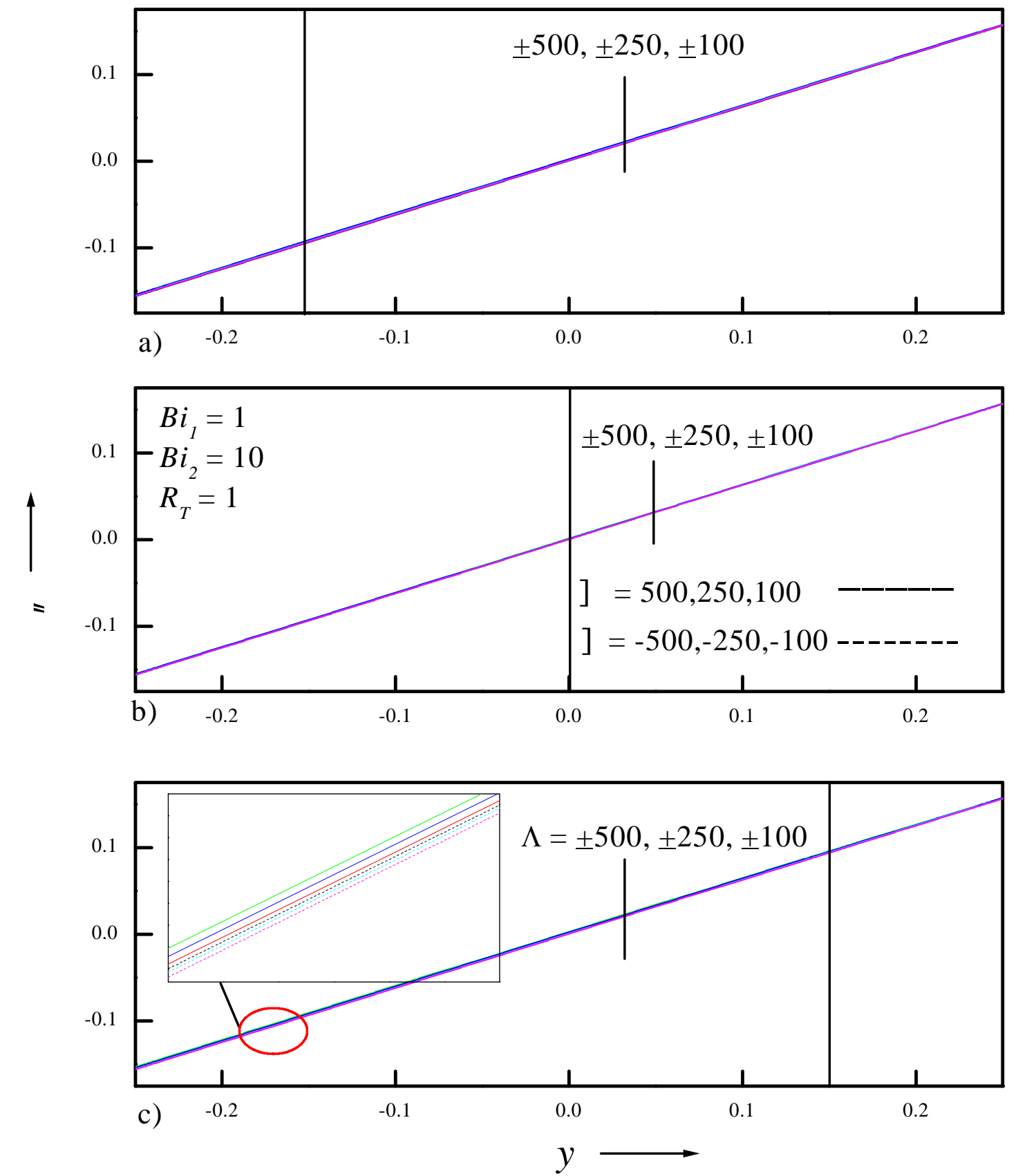

Figure 5: Temperature profiles for different values of $\Lambda$
a) $y^{*}=-0.15$,
b) $y^{*}=0.0$
c) $y^{*}=0.15$. 

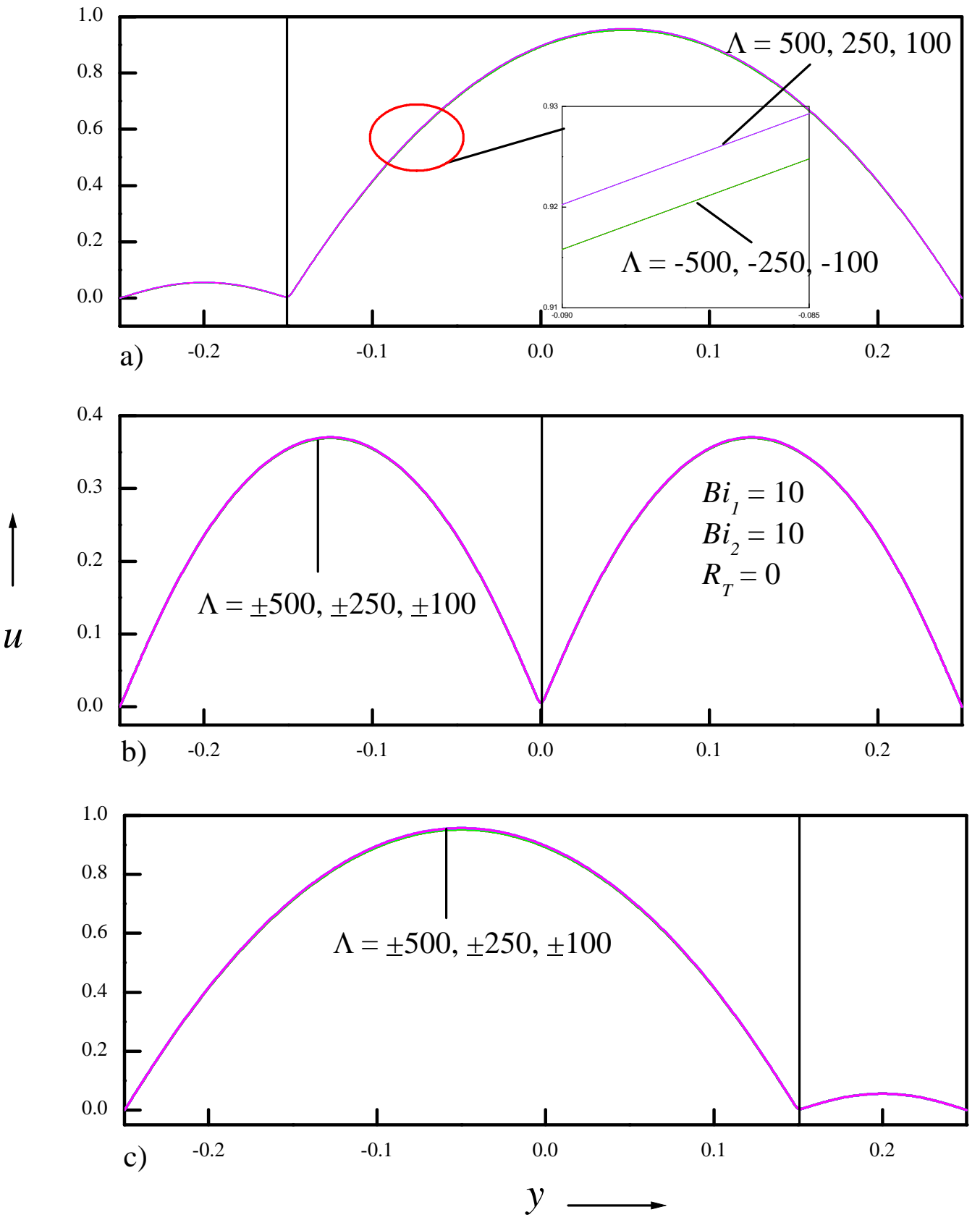

Figure 6: Velocity profiles for different values of $\Lambda$
a) $y^{*}=-0.15$,
b) $y^{*}=0.0$
c) $y^{*}=0.15$. 

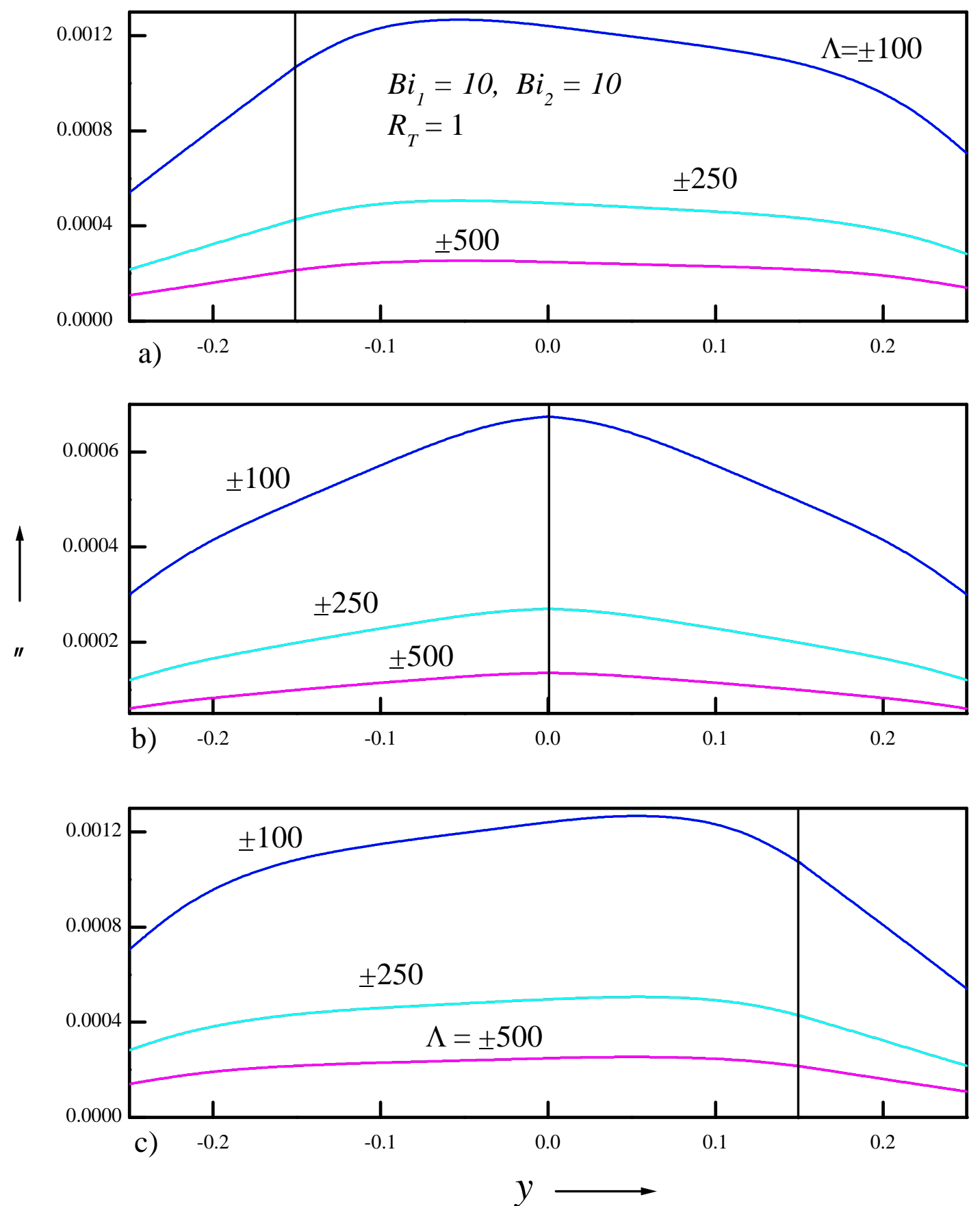

Figure 7: Temperature profiles for different values of $\Lambda$ at
a) $y^{*}=-0.15$,
b) $y^{*}=0.0$
c) $y^{*}=0.15$. 

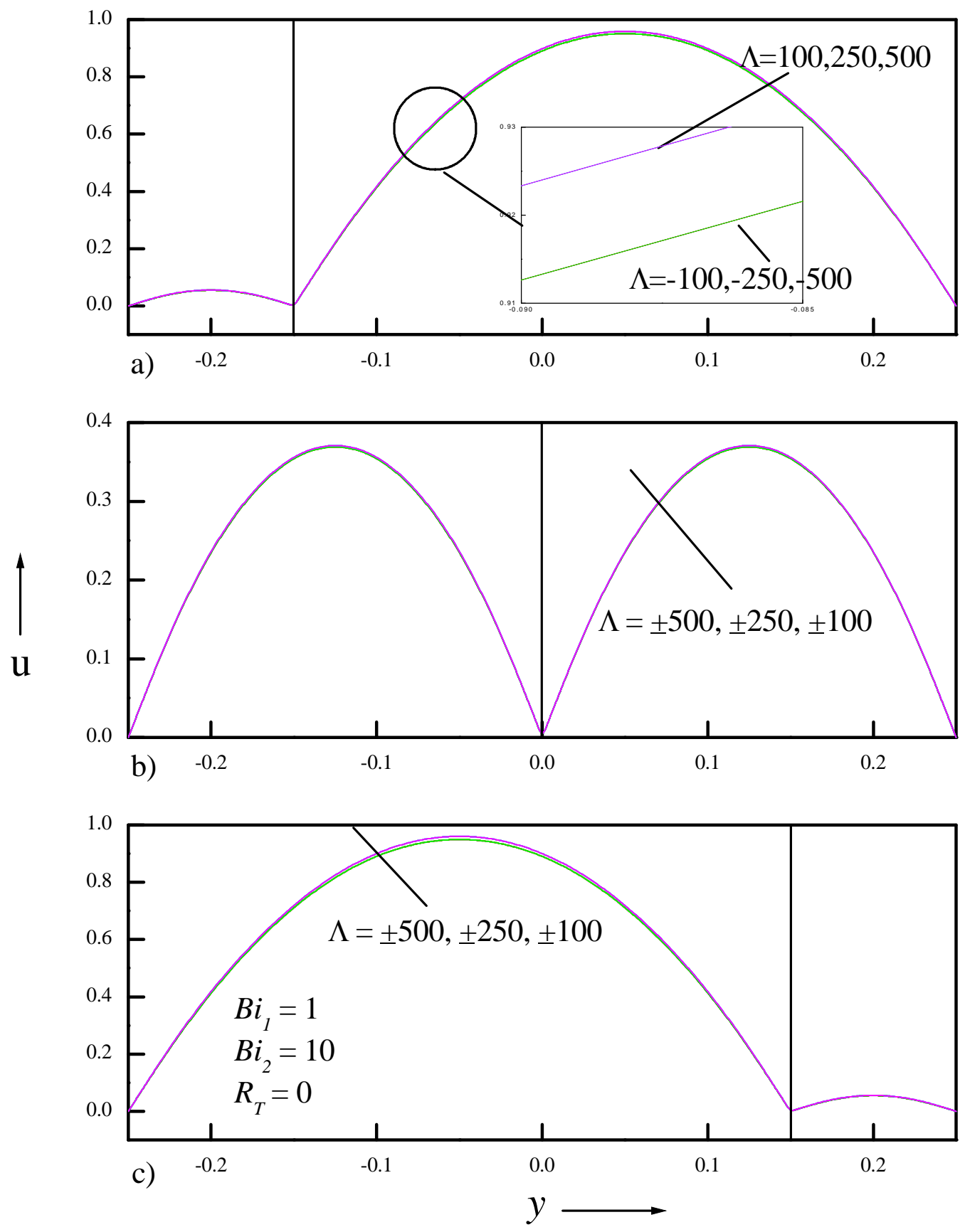

Figure 8: Velocity profiles for different values of $\Lambda$
a) $y^{*}=-0.15$,
b) $y^{*}=0.0$
c) $y^{*}=0.15$. 

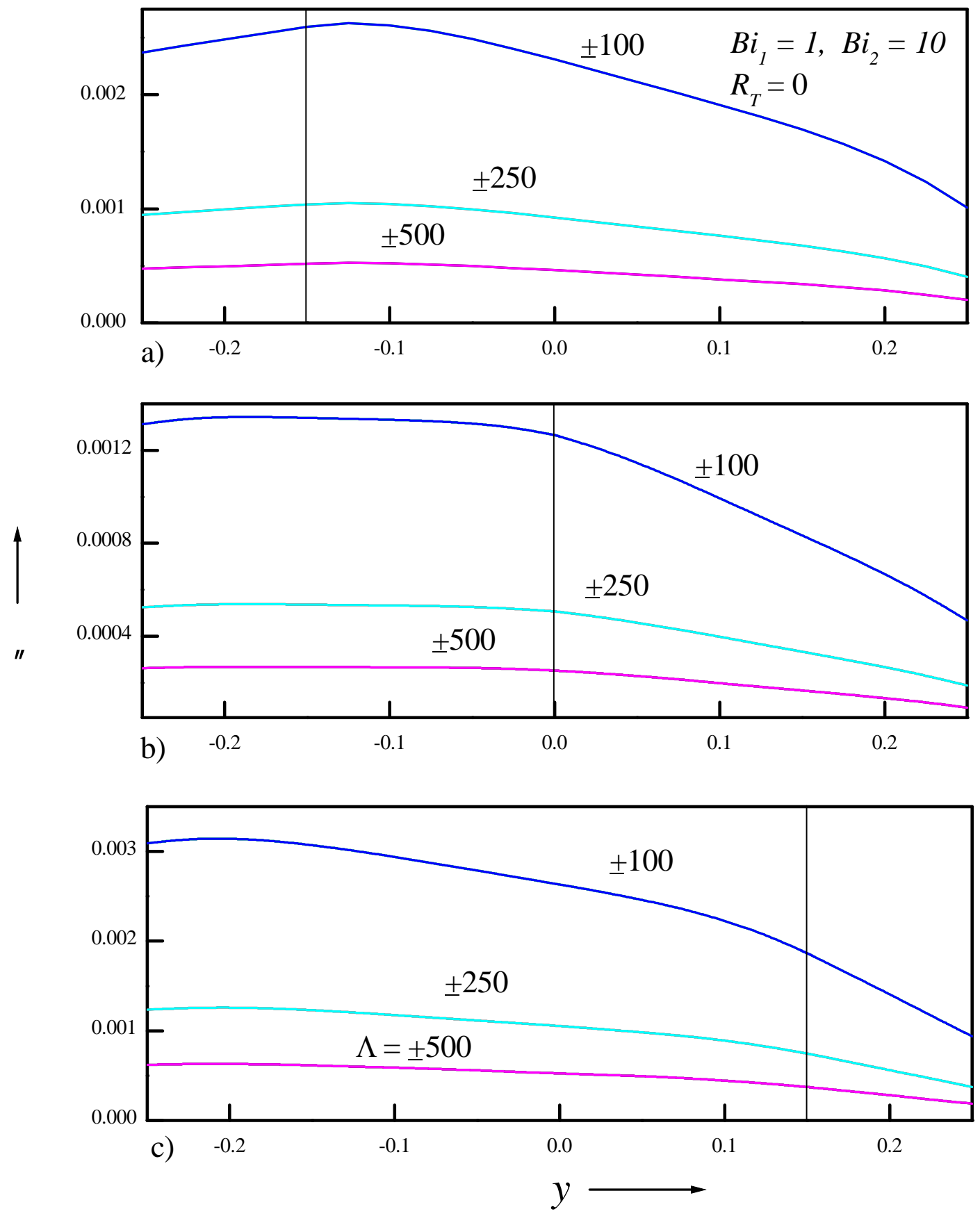

Figure 9: Temperature profiles for different values of $\Lambda$ at
a) $y^{*}=-0.15$,
b) $y^{*}=0.0$
c) $y^{*}=0.15$. 

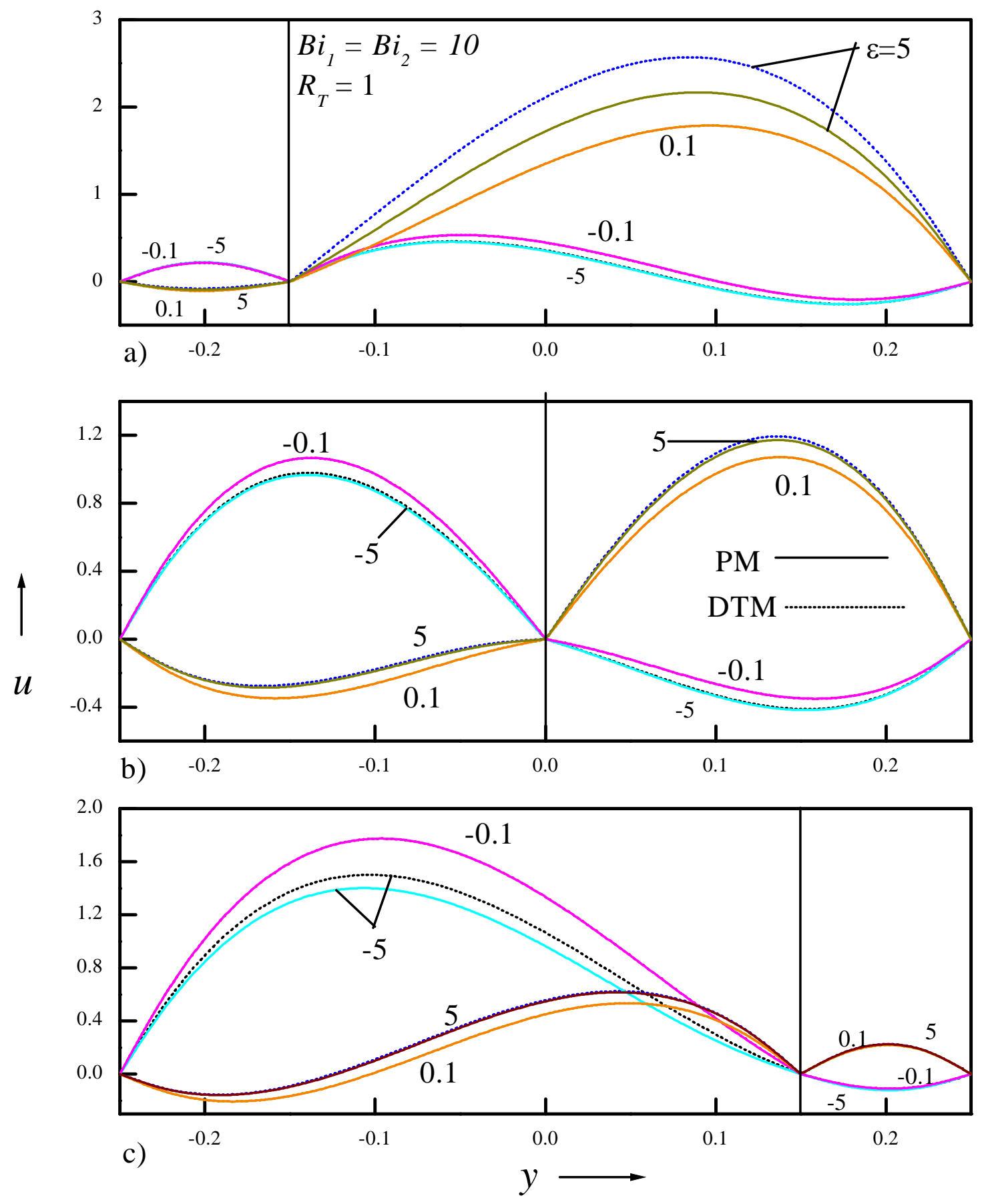

Figure 10: Velocity profiles for different values of $\varepsilon$ and $\Lambda$ at
a) $y^{*}=-0.15$,
b) $y^{*}=0.0$
c) $y^{*}=0.15$. 

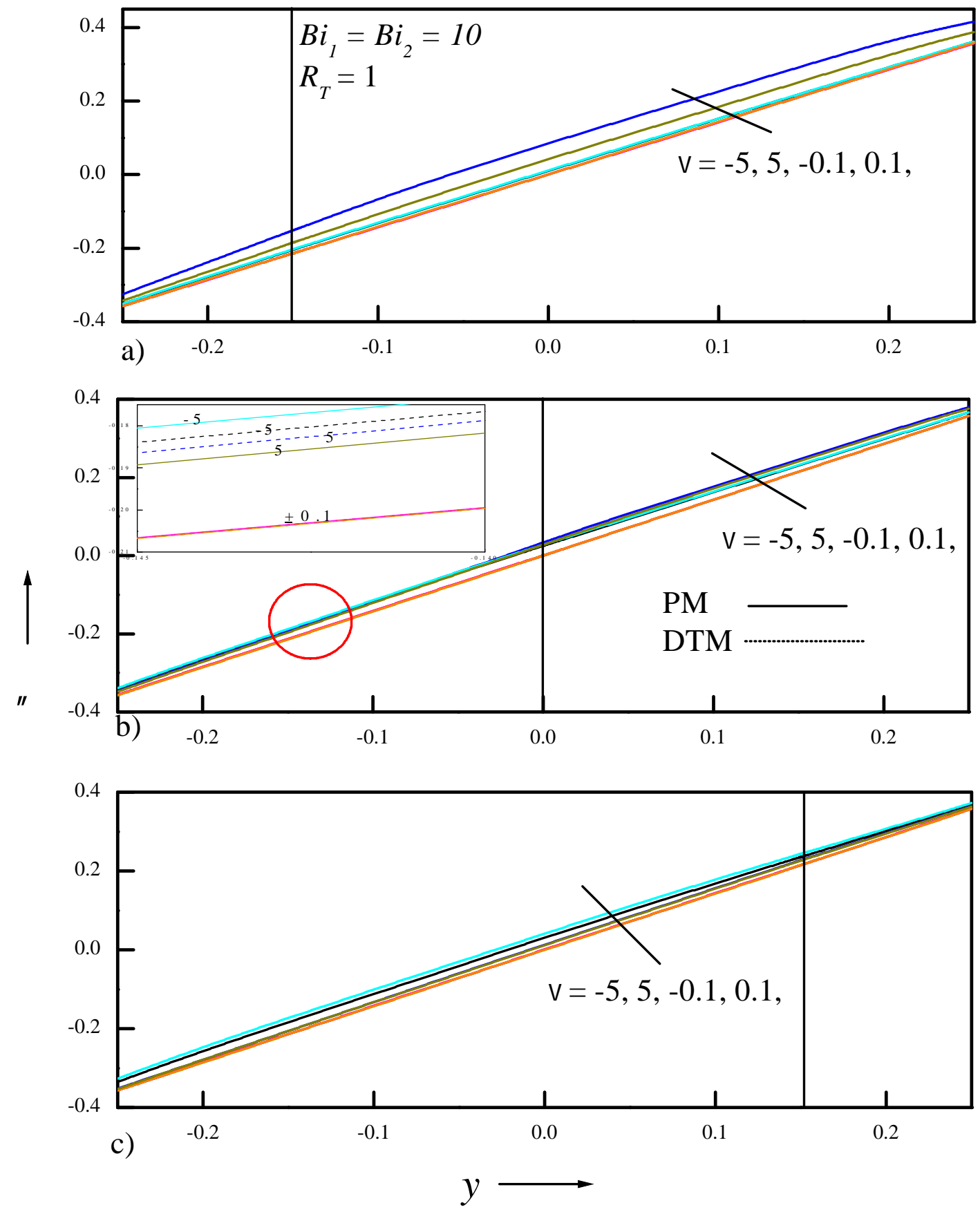

Fig.11. Temperature profiles for different values of $\varepsilon$ and $\Lambda$ at a) $y^{*}=-0.15, \quad$ b) $y^{*}=0.0$ c) $y^{*}=0.15$.

In order to know the flow nature for large values of $\varepsilon$, the solutions are obtained using DTM and shown in Figures 10 and 11 for asymmetric wall heating and for equal Biot numbers. It is seen from these figures that for upward flow $(\varepsilon>0)$ velocity and temperature are increasing functions of $\varepsilon$ and for downward flow $(\varepsilon<0)$ velocity is a decreasing function of $\varepsilon$ and temperature is an increasing function of $\varepsilon$. One can also conclude from Figures 10 and 11 that the solutions obtained by DTM and PM agree very well for values $\varepsilon<1$ and the error increases as $\varepsilon$ increases. The effects of mixed convection parameter $\Lambda$ on the flow for equal and unequal Biot numbers for $\varepsilon= \pm 0.1$ is the similar result observed by Zanchini (1998) in the absence of baffle. 
To understand the physical significance, the rate of heat transfer is evaluated for variations of mixed convection parameter, perturbation parameter and Biot numbers at different positions of the baffle at both the plates. It is seen that as the perturbation parameter $\varepsilon$, and the Biot numbers increases $\left(B i_{1}, B i_{2}\right)$ rate of heat transfer increases and it decreases as the mixed convection parameter $\Lambda(\Lambda>0)$ increases at the left plate at all the baffle positions. The rate of heat transfer increases as $\Lambda(\Lambda>0)$ increases at $y *=-0.15$ and $y *=0.15$ and decreases slightly $y *=0.0$ at the right plate. As the perturbation parameter $\varepsilon$ increases rate of heat transfer decreases whereas the reversal effect is observed for increasing values of Biot numbers at all the baffle positions at the right plate.

\section{Conclusion}

The effect of mixed convection parameter, equal and unequal Biot numbers and perturbation parameter using Robin boundary conditions in a vertical double passage channel was analysed. The solutions were found using regular perturbation method and differential transform method. For buoyancy assisting flow, the velocity increases in stream-II and for buoyancy opposing flow, the velocity increases in stream-I at all baffle positions. The velocity and temperature increases as the perturbation parameter increases for buoyancy assisting flow whereas velocity increases, temperature increases for buoyancy opposing flow at all the baffle positions. Temperature profiles were symmetric for equal Biot numbers and becomes stronger near the left plate for small values of $B i_{1}$. The Nusselt number increases at the left plate and decreases at the right plate as the perturbation parameter increases, whereas it increases at both the plates for increasing Biot numbers. The solutions obtained by the perturbation method and differential transform method were close for small values of the perturbation method. The results agreed very well with Zanchini (1998) in the absence of baffle. It is observed that there is an enhancement of the heat transfer in the channel by introducing the baffle and hence lot of research work can be done for the convection flow problems by introducing baffles.

\section{Acknowledgment}

One of the authors, J.C. Umavathi, is thankful for the financial support under the UGC-MRP F.43-66/2014 (SR) Project and also to Prof. Maurizio Sasso, supervisor and Prof. Matteo Savino co-coordinator for their support to do Post-Doctoral research under the scheme of ERUSMUS MUNDUS "Featured eUrope and South/south-east Asia mobility Network FUSION"

\section{Nomenclature}

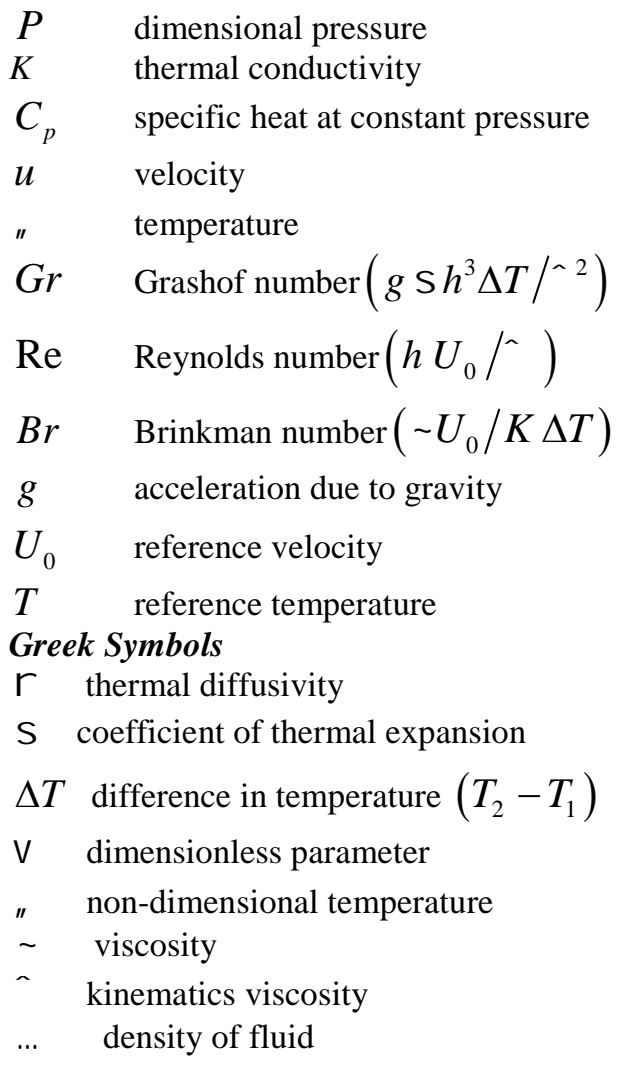




\section{References}

Aung, W., Worku, G., 2008. Theory of fully developed combined convection including flow reversal, ASME J. Heat Transfer, Vol.108, pp. 485-488.

Barletta, A., 1998. Laminar mixed convection with viscous dissipation in a vertical channel, Int. J. Heat and Mass Transfer, Vol. 41, pp. 3501-3513.

Umavathi, J.C., Mallikarjun B Patil, Pop I., 2006. On laminar mixed convection flow in a vertical porous stratum with symmetric wall heating conditions, Int. J. Trans. Phenom, Vol. 8, pp.127-140.

Umavathi, J.C., 2011. Mixed convection of micro polar fluid in a vertical double-passage channel, Int. J. Eng. Sci. Techno., Vol. 3, pp.197-209.

Umavathi, J.C., Jaweriya Sultana, 2013. Mixed convection flow in a vertical channel filled with porous medium with boundary conditions of third kind with heat source/sink-Brinkman source/sink-Brinkman model, Int. J. Math. Archive, Vol. 4, pp. 294-314.

Prathap Kumar, J., Umavathi, J.C., Pop, I., Basavaraj M Biradar, 2009. Fully developed mixed convection flow in a vertical channel containing porous and fluid layers with isothermal or isoflux boundaries, Trans. Porous Media, Vol. 80, pp. $117-135$.

Prathap Kumar, J., Umavathi, J.C., Basavaraj M Biradar, 2010. Mixed convection of composite porous medium in a vertical channel with asymmetric wall heating conditions, J. Porous Media, Vol. 13, pp. 271-285.

Prathap Kumar, J., Umavathi, J.C., Basavaraj M Biradar, 2011. Mixed convection of magneto hydrodynamic and viscous fluid in a vertical channel, Int. J. Non-Linear Mech., Vol. 46, pp. 278-285.

Angirasa, D., 2000. Mixed convection in a vertical enclosure with an isothermal vertical surface, Fluid Dynamics Research, Vol. 26, pp. 219-233.

Sumon Saha, Goutam Saha, Mohammed Ali, Md. Quamrul Islam, 2006. Combined free and forced convection inside a two dimensional multiple ventilated rectangular enclosure, ARPN Journal of Engineering and Applied Sciences., Vol. 1, pp. 2325.

Salah El-Din, M.M., 1994. Fully developed laminar convection in a vertical double passage channel, Appl. Energy, Vol. 47, pp. 69-75.

Dutta, P., Dutta, S., 1998. Effect of baffle size perforation and orientation on internal heat transfer enhancement, Int. J. Heat Mass Transfer, Vol. 41, pp. 3005-3013.

Chen, Z.D., Chen, J.J.J., 1998. Local heat transfer for oscillatory flow in the presence of a single baffle within a channel, Chem. Eng. Sci., Vol. 53, pp. 3177-3180.

Chang, T.S., Shiau, Y.H., 2005. Flow pulsation and baffle's effects on the opposing mixed convection in a vertical channel, Int. J. Heat Mass Transfer, Vol. 48, pp. 4190-4204.

Umavathi, J.C., Liu, I.C., Ali J. Chamkha, 2014. Mixed convection flow in a vertical channel filled with a fluid-saturated porous medium divided by a perfectly conductive baffle, Int. J. Microscale and Nanoscale, Vol. 5, pp. 127-147.

Prathap Kumar, J., Umavathi, J.C., Ali J. Chamkha, Prema, H., 2011a. Free convection in a vertical double passage wavy channel filled with a walter's fluid (model B), Int. J. Energy and Technology, Vol. 3, pp. 1-13.

Prathap Kumar, J., Umavathi, J.C., Prema, H., 2011b. Free convection of walter's fluid flow in a vertical double-passage wavy channel with heat source, Int. J. Eng. Sci. Techno., Vol. 3, pp. 136-165.

Umavathi, J.C., 2011, Mixed convection of micropolar fluid in a vertical double-passage channel, Int. J. Eng. Sci. Techno., Vol. 3, pp. 197-209.

Javeri, V., 1976. Analysis of laminar thermal entrance region of elliptical and rectangular channels with Kantorowich method, Warme-und Stoffuberragung, Vol. 9, pp. 85-98.

Sparrow, E.M., Siegal, R., 1960. Application of variation methods to the thermal entrance region of ducts, Int. J. Heat Mass Transfer, Vol. 1, pp. 161-172.

Javeri, V., 1977. Heat transfer in laminar entrance region of a flat channel for the temperature boundary condition of the third kind, Warme-und Stoffuberragung, Vol. 10, pp. 137-144.

Javeri, V., 1978. Laminar heat transfer in a rectangular channel for the temperature boundary condition of the third kind, Int. J. Heat Mass Transfer, Vol. 10, pp. 1029-1034.

Zanchini, E., 1998. Effect of viscous dissipation on mixed convection in a vertical channel with boundary conditions of the third kind, Int. J. Heat and Mass Transfer, Vol. 41, pp. 3949-3959.

Umavathi, J.C., Santosh Veershetty, 2012a. Non-Darcy mixed convection in a vertical porous channel with boundary conditions of third kind, Transport in Porous Media, Vol. 95, pp. 111-131.

Umavathi, J.C., Santosh Veershetty, 2012b. Mixed convection of a permeable fluid in a vertical channel with boundary conditions of third kind, Heat Transfer-Asian Research, Vol. 41, pp. 516-535.

Umavathi, J.C., Jaweriya Sultana, 2011a. Mixed convection of a micropolar fluid in a vertical channel with boundary conditions of third kind, Int. J. Eng. Sci. Techno., Vol. 3, pp. 213-224.

Umavathi, J.C., Jaweriya Sultana, 2011b. Mixed convection flow of conducting fluid in a vertical channel with boundary conditions of third kind in the presence of heat source/sink, Int. J. Energy and Technology, Vol. 3, pp. 1-10. 
Umavathi, J.C., Prathap Kumar, J., Jaweriya Sultana, 2012. Mixed convection flow in a vertical channel with boundary conditions of the third kind in the presence of heat source/sink, Appl. Math. Mech. , Vol. 33, pp. 1015-1034.

Sivasankaran, S., Kandaswamy, P., 2007. Effect of conductive baffle on hot-wall in double diffusive convection of water near density maximum, The Arabian Journal for Science and Engineering, Vol. 32, pp. 35-48.

Hajmohammadi, M.R., Nourazar, S.S., 2014. On the insertion of a thin gas layer in micro-cylindrical Couette flows involving power-law liquids, Int. J. Heat and Mass Transfer, Vol. 75, pp. 97-108

Zhou, J.K., 1986. Differential transformation and its applications for electrical circuits (in Chinese), Huazhong University Press, Wuhan, China.

\section{Biographical notes}

J. C. Umavathi received Ph. D degree from Gulbarga University Gulbarga India in 1992. She is a Professor in the department of Mathematics, Gulbarga University, Gulbarga, Karnataka, India. Her research interest includes heat and mass transfer of multiple (Newtonian and non-Newtonian) fluids through channels and rectangular ducts, numerical simulation using Finite differences and Runge-Kutta Gill method, magnetohydradynamics, flow through porous media she has published more than 60 papers in referred International journals. She has also presented more than 20 research articles in National and International conferences. She is currently dealing with few projects sponsored by Government of India.

J. Prathap Kumar received M. Phil. degree from Mangalore University Mangalore in 1990 and Ph. D degree from Gulbarga University Gulbarga India in 2003. $\mathrm{He}$ is a associate professor in the department of Mathematics Gulbarga University, Gulbarga, Karnataka, India. His research interest includes heat and mass transfer, dispersion, baffles for flow through various geometries of one and two fluids models. He has published more than 20 papers in referred International journals. He has also presented 5 research articles in national conferences.

M. Karuna Prasad is with the Department of Mathematics, Gulbarga University, Kalaburagi-585 106, Karnataka, India

Received January 2016

Accepted July 2016

Final acceptance in revised form August 2016 